\title{
The (Un)identification of Continuous-Action Rational Inattention Decision Models
}

\author{
Rongyu Wang* \\ Independent Researcher (PhD Economics, University of Edinburgh, 2016), Qingdao, 266072, China
}

\begin{abstract}
Cite This Paper in the following Citation Styles
(a): [1] Rongyu Wang, "The (Un)identification of Continuous-Action Rational Inattention Decision Models," Advances in Economics and Business, Vol.9, No.1, pp. 11-21, 2021. DOI: 10.13189/aeb.2021.090102

(b): Rongyu Wang, (2021). The (Un)identification of Continuous-Action Rational Inattention Decision Models. Advances in Economics and Business, 9(1), 11-21. DOI: 10.13189/aeb.2021.090102
\end{abstract}

Copyright @2021 by authors, all rights reserved. Authors agree that this article remains permanently open access under the terms of the Creative Commons Attribution License 4.0 International License

\begin{abstract}
In this paper, we discuss some properties of optimal consideration set in binary state continuous-action rational inattention decision problems. First, we show that it is the form of the utility function that determines the form of posterior distribution, i.e. whether it is a binary distribution or a triple or quadruple distribution, etc. Besides, we will also show that the posterior distribution may not be resulted after information acquisition in some circumstances. Second, we show that the optimal consideration set is essentially the optimal action strategies. Therefore, without further conditions, the rational inattention decision model is unidentifiable. Third, in order to identify the rational inattention decision model, we introduce another condition to the model. The discrete actions after information acquisition that satisfy the endogenously-determined probability (optimal action strategies) should satisfy the properties of probabilities, and therefore the discrete actions should also follow an exogenous probability distribution. This idea is tested by an example with quadratic utility function. Through this example, we find that under this condition, the optimal consideration set can be identified. The elements in the optimal consideration set, or equivalently the discrete signals after information acquisition, exhibit a curve or curves.
\end{abstract}

Keywords Rational Inattention Theory, Optimal Consideration Set, Continuous Action, Identification

\section{Introduction}

Rational inattention theory studies economic agents' information acquisition activities. Due to limited cognitive ability, human beings cannot always pay full attention to every object they care (Kahneman [4]; Tutino [14]). In the discipline of cognitive psychology, it has been a long tradition to study cognitive activities as information acquisition activities. Therefore, rational inattention theory is particularly suitable to model bounded rationality caused by limited attention. ${ }^{1}$ Since Sims $[11,12,13]$, rational inattention theory has been significantly developed. It is not only studied in macroeconomics and behavioral economics, but also in Finance (Jung, Kim, Matějka, Sims [3]), etc.

In this paper, our discussion focuses on a critical concept in rational inattention literature - optimal consideration set. The concept optimal consideration set is proposed by Caplin, Dean and Leahy [1]. Jung, Kim, Matějka, Sims [3] obtained a similar result. In particular, for continuous-action rational inattention decision models, optimal consideration set implies that at optimum, only discrete actions will be adopted by the agents. For example, a price setter could have opted any price. However, after information acquisition, the price setter will only focus on two prices (Matějka [8]). Therefore, optimal consideration set is regarded as a result or condition to determine the decision makers' optimal actions. Accordingly, in empirical application, the core of estimating a continuousaction rational inattention model is estimating its optimal consideration set.

The present literature of econometrics researches on rational inattention theory focuses on discrete-action rational inattention models. For details on this area, please refer to Maćkowiak, Matějka and Wiederholt [6]. Our paper is the

\footnotetext{
${ }^{1}$ Therefore, information theory is widely used in the study of rational inattention theory. For specific knowledge about information theory, please refer to Shannon [10] and Cover and Thomas [2].
} 
first one that studies the econometrics on continuous-action rational inattention models, which also have wide applications as discrete-action rational inattention models as exhibited in the review by Maćkowiak, Matějka and Wiederholt [6]. In particular, we prove that unlike discrete-action rational inattention models, the continuous-action rational inattention decision model studied in our paper cannot be identified by itself. Its identification requires additional condition(s) which is beyond the typical specification of the rational inattention decision models as in Matějka and McKay [7] or Matějka [8].

We analytically discuss the properties of optimal consideration set based on a simple case - a binary state continuous-action rational inattention decision model and attempt to identify the optimal consideration set. Our purpose is to deepen our understanding of the continuous-action rational inattention decision model through the simple case. First, we find that it is the utility function that determines how many discrete signals or actions there are in the optimal consideration set after information acquisition. In particular, if we adopt a quadratic utility function, we can only get a binary posterior. Meanwhile, we find that under certain conditions, the optimal consideration set can be an empty set.

Second, we find that in the binary state continuous-action rational inattention decision model, the optimal consideration set is essentially the optimal action strategy. They are the same thing, and just because they are the same thing, it makes the signals after information acquisition actually cannot be identified from the optimal consideration set. If we want to identify the signals, at least for the binary state model, we need extra conditions.

Third, we derive an additional condition to identify the signals based on the probability properties the optimal signals or signals after information acquisition should follow. If the endogenously-determined probabilities represented by optimal action strategies are true probabilities, they should be subject to the properties of probabilities such that the optimal signals follows an exogenously specified probability distribution. We test this idea by adopting quadratic utility function as an example, and we find that with this condition, the signals in the optimal consideration set can be identified. The optimal consideration set is non-empty, and contrary to some conventional perceptions that the optimal consideration set just include several scattered points, we find that at least a subset of the optimal consideration set is a curve or curves, which indicates that the number of elements in the optimal consideration set is infinite.

This paper is organized as follows. Section 2 explains why in this model, utility function determines the form of posterior distribution. Section 3 explains why without extra conditions, the present rational inattention decision model cannot be identified. Section 4 proposes the new condition that is used to identify the rational inattention decision model. Section 5 concludes the paper.

\section{Utility Function Determines the Form of the Posterior Distribution.}

In this section, we will show that for the continuous action rational-inattention decision problem, given the prior distribution, the form of the utility function determines the form of the posterior distribution, i.e. whether the posterior distribution contains two discrete signals or three discrete signals. We will also show that the prior distribution in some situations cannot result in any posterior distribution. In particular, the proof below shows that for a certain type of continuous prior distribution, no matter what form of utility function it is, the rational inattention decision problem can result in an optimal action strategy in a binary form (i.e., a binary posterior). The approach we use to construct the rational inattention decision problem follows the two step approach in Matějka and McKay [7], and the terms here we use to describe the rational inattention decision problem is also consistent with the literature. We will not describe how we construct the rational-inattention decision problem here so that we can focus our attention on the core contents of the paper. Readers can refer to Matějka and McKay [7] or Matějka [7] to construct the rational inattention problem. In the following, we provide the primitive parameters that are used to construct the rational inattention decision problem.

The decision maker is able to get a prior distribution describing demand. Suppose the prior distribution follows a binary distribution. It is state $\varepsilon \in\{u, d\}, \operatorname{Pr}(u)=p$ and $\operatorname{Pr}(d)=1-p$. The action is the ordering quantity $\alpha \in \mathbb{R}$. The utility function takes the following quadratic form $u(\alpha, \varepsilon)=-(\alpha-\varepsilon)^{2}$. Given an underlying state $\varepsilon$, the decision maker acquires information about demand to choose the optimal ordering quantity $\alpha$ to maximize their utilities. The unit information processing cost is described by $\lambda$. The information is processed according to Shannon entropy. The action strategy is denoted by $f(\alpha \mid \theta)$. Applying Matějka and McKay's [7] two stage approach, we obtain the decision-making problem by:

$$
\begin{gathered}
\max _{f(\alpha \mid \theta)} \int_{-\infty}^{+\infty} \int_{-\infty}^{+\infty}[\Pi(f(u \mid \alpha)) f(\alpha \mid u) p+\Pi(f(d \mid \alpha)) f(\alpha \mid d)(1-p)] d \alpha \\
-\lambda I(f(\alpha \mid \varepsilon))
\end{gathered}
$$

s.t.

$$
\begin{gathered}
f(\alpha \mid \varepsilon) \geq 0 \\
\int_{-\infty}^{+\infty} f(\alpha \mid \varepsilon) d \alpha=1
\end{gathered}
$$

where

$$
\Pi(f(\varepsilon \mid \alpha)) \equiv \max _{\alpha}-(\alpha-u)^{2} \times p-(\alpha-d)^{2} \times(1-p)
$$




$$
I(f(\alpha \mid \varepsilon))=H(f(\alpha))-\mathbb{E}_{\varepsilon}[H(f(\alpha \mid \varepsilon))]
$$

where $H($.$) represents the Shannon entropy function.$

Solving this decision-making problem, we obtain the optimal action strategy by

$$
f(\alpha \mid \varepsilon)=\frac{f(\alpha) \exp \left(\frac{-(\alpha-\varepsilon)^{2}}{\lambda}\right)}{\int_{-\infty}^{+\infty} f(\alpha) \exp \left(\frac{-(\alpha-\varepsilon)^{2}}{\lambda}\right) d \alpha}
$$

where

$$
\begin{gathered}
\frac{p}{\int_{-\infty}^{+\infty} f(\alpha) \exp \left(\frac{-(\alpha-u)^{2}}{\lambda}\right) d \alpha} \exp \left(\frac{-(\alpha-u)^{2}}{\lambda}\right) \\
+\frac{1-p}{\int_{-\infty}^{+\infty} f(\alpha) \exp \left(\frac{-(\alpha-d)^{2}}{\lambda}\right) d \alpha} \exp \left(\frac{-(\alpha-d)^{2}}{\lambda}\right)=1
\end{gathered}
$$

which is obtained according to the probability property $\int_{-\infty}^{+\infty} f(\alpha \mid \theta) g(\theta) d \theta=f(\alpha)$.

Define the function $F(\alpha)$ by

$$
\begin{gathered}
F(\alpha)=\frac{p}{\int_{-\infty}^{+\infty} f(\alpha) \exp \left(-\frac{(\alpha-u)^{2}}{\lambda}\right) d \alpha} \exp \left(-\frac{(\alpha-u)^{2}}{\lambda}\right) \\
+\frac{1-p}{\int_{-\infty}^{+\infty} f(\alpha) \exp \left(-\frac{(\alpha-d)^{2}}{\lambda}\right) d \alpha} \exp \left(-\frac{(\alpha-d)^{2}}{\lambda}\right)
\end{gathered}
$$

According to Caplin, Dean and Leahy [1], the solutions of the equation $F(\alpha)=1$ belongs to the optimal consideration set. Naturally we will ask how many solutions there are in equation (1). For simplicity, define $W_{1} \equiv \frac{p}{\int_{-\infty}^{+\infty} f(\alpha) \exp \left(-\frac{(\alpha-u)^{2}}{\lambda}\right) d \alpha}$ and $W_{2} \equiv \frac{1-p}{\int_{-\infty}^{+\infty} f(\alpha) \exp \left(-\frac{(\alpha-d)^{2}}{\lambda}\right) d \alpha}$. Therefore, $F(\alpha)$ can be reformulated by $F(\alpha)=W_{1} \exp \left(-\frac{(\alpha-u)^{2}}{\lambda}\right)+W_{2} \exp \left(-\frac{(\alpha-d)^{2}}{\lambda}\right)$. Let's further define $D_{1}(\alpha)=W_{1} \exp \left(-\frac{(\alpha-u)^{2}}{\lambda}\right)$ and $D_{2}(\alpha)=1-W_{2}\left(\alpha_{1}, \alpha_{2}\right) \exp \left(-\frac{(\alpha-d)^{2}}{\lambda}\right)$. Therefore, for $\alpha \lessgtr u, \frac{d D_{1}(\alpha)}{d \alpha} \gtrless 0$, and for $\alpha \lessgtr d, \frac{d D_{2}(\alpha)}{d \alpha} \lessgtr 0$. According to these results, we can transform the problem of finding solutions to $F(\alpha)=1$ into finding the intersection points between $D_{1}(\alpha)$ and $D_{2}(\alpha)$. We can divide the question into four scenarios and discuss them specifically.

$$
\text { 2.1 } D_{1}(d)<D_{2}(d), D_{1}(u)>D_{2}(u)
$$

In Figure 1, according to the specification $D_{1}(d)<D_{2}(d)$, $D_{1}(u)>D_{2}(u), D_{1}(\alpha)$ and $D_{2}(\alpha)$ must have intersection points with each other, and the number of intersection points

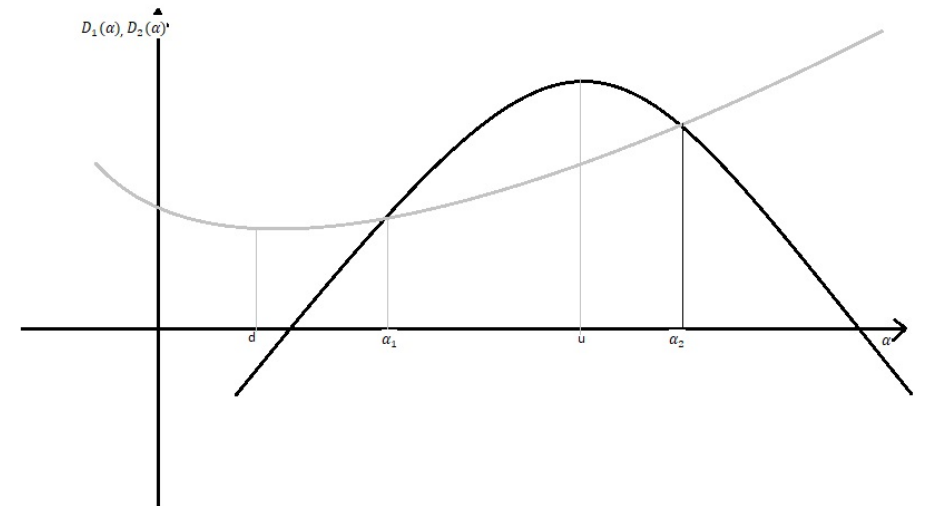

Figure 1: $D_{1}(\alpha)$ and $D_{2}(\alpha)$ when $D_{1}(d)<D_{2}(d)$, $D_{1}(u)>D_{2}(u)$. The black curve represents $D_{1}(\alpha)$. The gray curve represents $D_{2}(\alpha)$. The thin lines are auxiliary lines.

are 2. Therefore, in this case, $F(\alpha)=1$ have two solutions and hence the optimal consideration set contains two elements. We will obtain a binary-action posterior in this case. Besides, $d<\alpha_{1}<u<\alpha_{2}$.

$$
\text { 2.2 } D_{1}(d)>D_{2}(d), D_{1}(u)<D_{2}(u)
$$

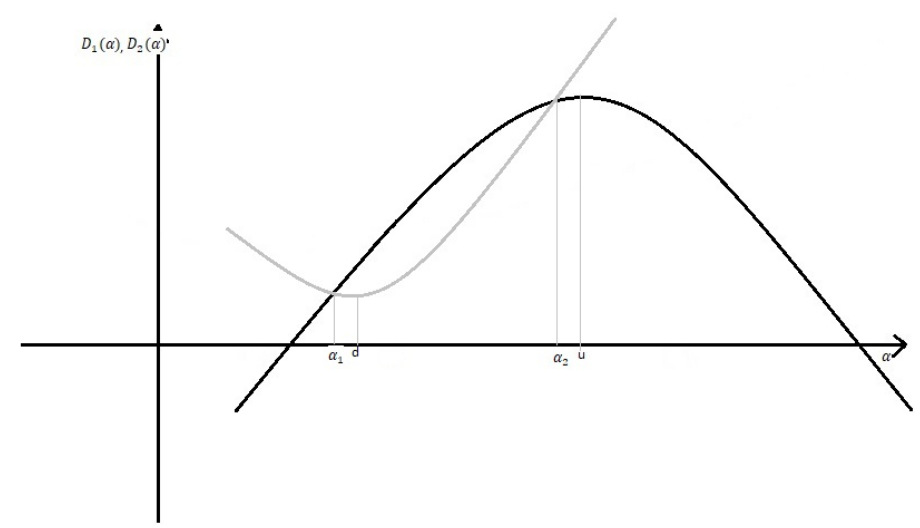

Figure 2: $D_{1}(\alpha)$ and $D_{2}(\alpha)$ when $D_{1}(d)>D_{2}(d)$, $D_{1}(u)<D_{2}(u)$. The black curve represents $D_{1}(\alpha)$. The gray curve represents $D_{2}(\alpha)$. The thin lines are auxiliary lines.

In Figure 2, according to the specification $D_{1}(d)>D_{2}(d)$, $D_{1}(u)<D_{2}(u), D_{1}(\alpha)$ and $D_{2}(\alpha)$ must have intersection points with each other, and the number of intersection points are 2. Therefore, in this case, $F(\alpha)=1$ have two solutions and hence the optimal consideration set contains two elements. We will obtain a binary-action posterior in this case. Besides, $\alpha_{1}<d<\alpha_{2}<u$.

\section{$2.3 D_{1}(d)>D_{2}(d), D_{1}(u)>D_{2}(u)$}

In Figure 3, according to the specification $D_{1}(d)>D_{2}(d)$, $D_{1}(u)>D_{2}(u), D_{1}(\alpha)$ and $D_{2}(\alpha)$ must have intersection points with each other, and the number of intersection points 


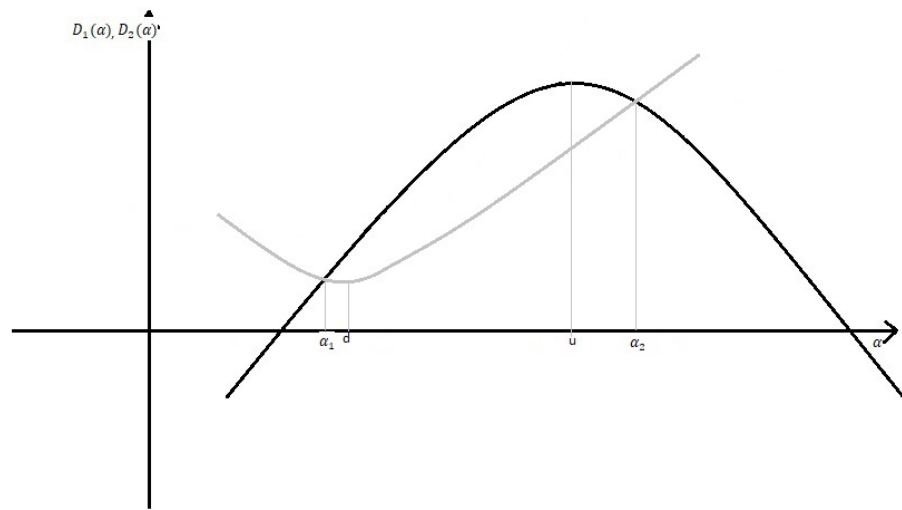

Figure 3: $D_{1}(\alpha)$ and $D_{2}(\alpha)$ when $D_{1}(d)>D_{2}(d)$, $D_{1}(u)>D_{2}(u)$. The black curve represents $D_{1}(\alpha)$. The gray curve represents $D_{2}(\alpha)$. The thin lines are auxiliary lines.

are 2. Therefore, in this case, $F(\alpha)=1$ have two solutions and hence the optimal consideration set contains two elements. We will obtain a binary-action posterior in this case. Besides, $\alpha_{1}<d<u<\alpha_{2}$.

\section{$2.4 D_{1}(d)<D_{2}(d), D_{1}(u)<D_{2}(u)$}

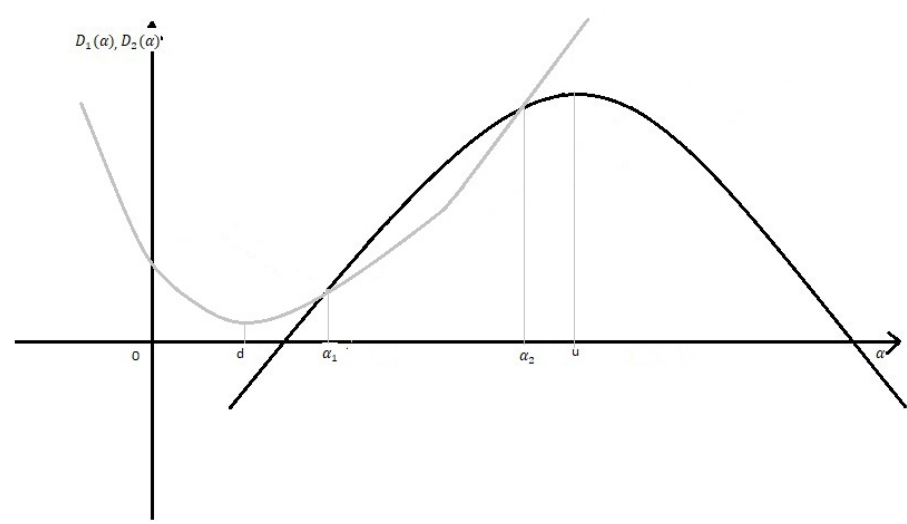

Figure 4: $D_{1}(\alpha)$ and $D_{2}(\alpha)$ when $D_{1}(d)<D_{2}(d)$, $D_{1}(u)<D_{2}(u)$. The black curve represents $D_{1}(\alpha)$. The gray curve represents $D_{2}(\alpha)$. The thin lines are auxiliary lines.

In Figure 4, according to the specification $D_{1}(d)<D_{2}(d)$, $D_{1}(u)<D_{2}(u), D_{1}(\alpha)$ and $D_{2}(\alpha)$ may not have intersection points with each other. Therefore, $F(\alpha)=1$ does not necessarily have solution. If $D_{1}(\alpha)$ and $D_{2}(\alpha)$ have intersection points, it could be either one or two. The one intersection point emerges when $D_{1}(\alpha)$ and $D_{2}(\alpha)$ are tangent to each other. The tangent point is just the intersection point in this case. However, remember that after information acquisition, the decision maker should obtain a probability distribution. Therefore, if there is only one intersection point, i.e. $F(\alpha)=1$ has a unique solution, supposing this point is chosen with probability one, then what points are chosen with probability zero? Therefore, a contradiction arises, and in this situation, $F(\alpha)=1$ still cannot result in a posterior distribution. Only when $D_{1}(\alpha)$ and $D_{2}(\alpha)$ have two intersection points, then a posterior distribution can be obtained.

Therefore, $F(\alpha)=1$ either has no solution or leads to a binary-action posterior. According to Figure 4, for $D_{1}(d)<D_{2}(d), D_{1}(u)<D_{2}(u)$, the condition to ensure a binary-action posterior exists is that for $\alpha \in(d, u)$, both $D_{1}(\alpha) \gtrless D_{2}(\alpha)$ happen. In this case, if we obtain a binaryaction posterior, then it follows that $d<\alpha_{1}<\alpha_{2}<1$.

Summarizing the four cases, we can find that $\alpha_{1}<u$, $\alpha_{2}>d$. Because the utility function is quadratic, therefore it determines that if $D_{1}(\alpha)$ and $D_{2}(\alpha)$ have intersection points, then the number of the intersection points is at most two, and therefore, we can obtain a binary-action posterior. If the utility function takes other firms, for example $a \alpha^{3}+b \alpha^{2}+c \alpha+d$, then there are at most three intersection points between $D_{1}(\alpha)$ and $D_{2}(\alpha)$. In this situation, we can obtain either a binary-action posterior, or a tripleaction posterior.

Proposition 1: Given a binary prior distribution on state in the continuous-action rational inattention decision problem, the form of the utility function determines what kind of posterior distribution on action we could obtain. If utility function is quadratic such that $u(\alpha, \varepsilon)=-(\alpha-\varepsilon)^{2}$, then the rational inattention decision problem, if it can result in a posterior, can only produce a binary-action posterior, i.e. the optimal consideration set only contains two elements.

Note that when $D_{1}(d)<D_{2}(d)$ and $D_{1}(u)<D_{2}(u)$, $D_{1}(u)$ and $D_{2}(u)$ may not have intersection points and this feature is seemingly determined by the properties of $D_{1}(\alpha)$ and $D_{2}(\alpha)$, but is essentially determined by the utility function and $\lambda$ as well as the prior distribution. Therefore, in our case, given the prior distribution and unit information acquisition cost, the utility function is also responsible for the non-existence of solutions to $F(\alpha)=1$.

Proposition 2: For the case where $D_{1}(d)<D_{2}(d)$ and $D_{1}(u)<D_{2}(u)$, if for all $\alpha \in(d, u), D_{1}(\alpha)<D_{2}(\alpha)$, then $F(\alpha)=1$ have no solution, i.e. the information acquisition is null.

Because the posterior distribution can only be a binary distribution, we can re-write the function $F(\alpha)$ by

$$
\begin{aligned}
& F(\alpha)=\frac{p}{q \exp \left(-\frac{\left(\alpha_{1}-u\right)^{2}}{\lambda}\right)+(1-q) \exp \left(-\frac{\left(\alpha_{2}-u\right)^{2}}{\lambda}\right)} \exp \left(-\frac{(\alpha-u)^{2}}{\lambda}\right) \\
& +\frac{1-p}{q \exp \left(-\frac{\left(\alpha_{1}-d\right)^{2}}{\lambda}\right)+(1-q) \exp \left(-\frac{\left(\alpha_{2}-d\right)^{2}}{\lambda}\right)} \exp \left(-\frac{(\alpha-d)^{2}}{\lambda}\right)
\end{aligned}
$$

$q$ is the probability of action $\alpha_{1}$ is chosen, i.e. $q=f\left(\alpha_{1}\right)=\operatorname{Pr}\left(\alpha_{1}\right)$, while $1-q$ is the probability of action $\alpha_{2}$ is chosen, i.e. $1-q=f\left(\alpha_{2}\right)=\operatorname{Pr}\left(\alpha_{2}\right)$. $\alpha_{1}$ and $\alpha_{2}$ are elements in the optimal consideration set $\left\{\alpha_{1}, \alpha_{2}\right\}$, or 
the solutions to $F(\alpha)=1 . \alpha_{1}$ and $\alpha_{2}$ correspond to the two discrete signals after information acquisition.

$$
\begin{aligned}
& \text { Accordingly, } W_{1}=\frac{p}{q \exp \left(-\frac{\left(\alpha_{1}-u\right)^{2}}{\lambda}\right)+(1-q) \exp \left(-\frac{\left(\alpha_{2}-u\right)^{2}}{\lambda}\right)} \\
& \text { and } W_{2}=\frac{1-p}{q \exp \left(-\frac{\left(\alpha_{1}-d\right)^{2}}{\lambda}\right)+(1-q) \exp \left(-\frac{\left(\alpha_{2}-d\right)^{2}}{\lambda}\right)} .
\end{aligned}
$$

Besides, the optimal ordering strategy $f(\alpha \mid \varepsilon)$ can be written into

$$
q^{\varepsilon}=\frac{q \exp \left(\frac{-(\alpha-\varepsilon)^{2}}{\lambda}\right)}{q \exp \left(\frac{-\left(\alpha_{1}-\varepsilon\right)^{2}}{\lambda}\right)+(1-q) \exp \left(\frac{-\left(\alpha_{2}-\varepsilon\right)^{2}}{\lambda}\right)}
$$

where $q^{\varepsilon}=f\left(\alpha_{1} \mid \varepsilon\right)=\operatorname{Pr}\left(\alpha_{1} \mid \varepsilon\right), 1-q^{\varepsilon}=f\left(\alpha_{2} \mid \varepsilon\right)=$ $\operatorname{Pr}\left(\alpha_{2} \mid \varepsilon\right)$.

Substitute $q^{\varepsilon}$ back into the objective function of the rational inattention decision problem, and we can obtain a new decision problem to obtain the optimal $q$ :

$$
\begin{aligned}
& \max _{q} \lambda\left\{p \ln \left[q \exp \left(\frac{-\left(\alpha_{1}-u\right)^{2}}{\lambda}\right)+(1-q) \exp \left(\frac{-\left(\alpha_{2}-u\right)^{2}}{\lambda}\right)\right]\right. \\
& \left.+(1-p) \ln \left[q \exp \left(\frac{-\left(\alpha_{1}-u\right)^{2}}{\lambda}\right)+(1-q) \exp \left(\frac{-\left(\alpha_{2}-u\right)^{2}}{\lambda}\right)\right]\right\}
\end{aligned}
$$

$$
\text { s.t } \quad 0 \leq q \leq 1
$$

we can obtain the interior solution to the optimal action strategy $q$, which is unique, by

$$
q=\frac{p}{1-\exp \left(\frac{-\left(\alpha_{1}-d\right)^{2}+\left(\alpha_{2}-d\right)^{2}}{\lambda}\right)}+\frac{1-p}{1-\exp \left(\frac{-\left(\alpha_{1}-u\right)^{2}+\left(\alpha_{2}-u\right)^{2}}{\lambda}\right)}
$$

$$
\frac{\underset{p}{\operatorname{supposing}} q \in[0,1] . \quad \text { The generic }}{\frac{1-p}{1-\exp \left(\frac{-\left(\alpha_{1}-d\right)^{2}+\left(\alpha_{2}-d\right)^{2}}{\lambda}\right)}+\frac{1}{1-\exp \left(\frac{-\left(\alpha_{1}-u\right)^{2}+\left(\alpha_{2}-u\right)^{2}}{\lambda}\right)} \text { could }}
$$
be greater than 0 or smaller than 0 . If the generic $q<0$, which is obtained from solving the decision problem without considering the constraint, the rational inattention decision problem needs to take a corner solution, which is $q=0$. Similarly, if the generic $q>1$, it also needs to take a corner solution, which is $q=1$.

The derivation of $f(\alpha \mid \varepsilon)$ and $q$ follows the standard steps in rational inattention literature. We will not address them in the paper. Interested readers can derive these action strategies by themselves as an exercise.
Now we obtain the explicit form of the optimal action strategy, which is expressed by equation (3). Together with $F(\alpha)=1$, it seems that we can proceed to identify the elements $\alpha_{1}$ and $\alpha_{2}$ in the optimal consideration set. However, in the following, we shall prove that the interior optimal action strategy $q$ is essentially the optimal consideration set. They are the same thing. Therefore, without additional condition, the elements in the optimal consideration sets or the discrete signals after information acquisition are unidentifiable.

\section{Optimal Consideration Set and Opti- mal Action Strategies are essentially the Same Thing.}

We call the elements in the optimal consideration set by optimal actions for continuous-action rational inattention decision problems. We find that for 2-optimal action and binary state continuous-action rational inattention decision problems, for interior solutions of the optimal action strategy, i.e. $q \in[0,1]$, the optimal consideration set is essentially the optimal action strategy. The following proof follows the utility function with the form $-(\alpha-\varepsilon)^{2}$. In fact, the proof applies to any form of utility function and the proof results are the same.

Recall equation (2). If $\alpha=\alpha_{1}$, then

$$
\begin{gathered}
\frac{p \exp \left(-\frac{\left(\alpha_{1}-u\right)^{2}}{\lambda}\right)}{q\left[\exp \left(-\frac{\left(\alpha_{1}-u\right)^{2}}{\lambda}\right)-\exp \left(-\frac{\left(\alpha_{2}-u\right)^{2}}{\lambda}\right)\right]+\exp \left(-\frac{\left(\alpha_{2}-u\right)^{2}}{\lambda}\right)}-p \\
=(1-p) \\
-\frac{(1-p) \exp \left(-\frac{\left(\alpha_{1}-d\right)^{2}}{\lambda}\right)}{q\left[\exp \left(-\frac{\left(\alpha_{1}-d\right)^{2}}{\lambda}\right)-\exp \left(-\frac{\left(\alpha_{2}-d\right)^{2}}{\lambda}\right)\right]+\exp \left(-\frac{\left(\alpha_{2}-d\right)^{2}}{\lambda}\right)}
\end{gathered}
$$

which can be reformulated to

$$
\begin{gathered}
\frac{p\left[\exp \left(-\frac{\left(\alpha_{1}-u\right)^{2}}{\lambda}\right)-\exp \left(-\frac{\left(\alpha_{2}-u\right)^{2}}{\lambda}\right)\right](1-q)}{q\left[\exp \left(-\frac{\left(\alpha_{1}-u\right)^{2}}{\lambda}\right)-\exp \left(-\frac{\left(\alpha_{2}-u\right)^{2}}{\lambda}\right)\right]+\exp \left(-\frac{\left(\alpha_{2}-u\right)^{2}}{\lambda}\right)} \\
=-\frac{p\left[\exp \left(-\frac{\left(\alpha_{1}-d\right)^{2}}{\lambda}\right)-\exp \left(-\frac{\left(\alpha_{2}-d\right)^{2}}{\lambda}\right)\right](1-q)}{q\left[\exp \left(-\frac{\left(\alpha_{1}-d\right)^{2}}{\lambda}\right)-\exp \left(-\frac{\left(\alpha_{2}-d\right)^{2}}{\lambda}\right)\right]+\exp \left(-\frac{\left(\alpha_{2}-d\right)^{2}}{\lambda}\right)}
\end{gathered}
$$

We do not consider the corner solution $q=1$ here. Therefore, the above equation can be reformulated by

$$
\begin{gathered}
q\left[\exp \left(-\frac{\left(\alpha_{1}-u\right)^{2}}{\lambda}\right)\right. \\
\left.-\exp \left(-\frac{\left(\alpha_{2}-u\right)^{2}}{\lambda}\right)\right]\left[\exp \left(-\frac{\left(\alpha_{1}-d\right)^{2}}{\lambda}\right)\right. \\
\left.-\exp \left(-\frac{\left(\alpha_{2}-d\right)^{2}}{\lambda}\right)\right]
\end{gathered}
$$


$=-p \exp \left(-\frac{\left(\alpha_{2}-d\right)^{2}}{\lambda}\right)\left[\exp \left(-\frac{\left(\alpha_{1}-u\right)^{2}}{\lambda}\right)-\exp \left(-\frac{\left(\alpha_{2}-u\right)^{2}}{\lambda}\right)\right]$

$-(1-p) \exp \left(-\frac{\left(\alpha_{2}-u\right)^{2}}{\lambda}\right)\left[\exp \left(-\frac{\left(\alpha_{1}-d\right)^{2}}{\lambda}\right)-\exp \left(-\frac{\left(\alpha_{2}-d\right)^{2}}{\lambda}\right.\right.$ The obtained $q$, if it belongs to [0, 1$]$, is exactly the interibr solution of optimal action strategy we obtained in last section by solving the rational inattention decision problem. Therefore, for interior solution to $q$, the optimal consideration

and hence set is essentially the optimal action strategy. Even when we consider the corner solutions, we will draw the same conclusion (the proof is simple and interested readers can show it by themselves).

$q=\frac{p}{1-\exp \left(\frac{-\left(\alpha_{1}-d\right)^{2}+\left(\alpha_{2}-d\right)^{2}}{\lambda}\right)}+\frac{1-p}{1-\exp \left(\frac{-\left(\alpha_{1}-u\right)^{2}+\left(\alpha_{2}-u\right)^{2}}{\lambda}\right)}$

If $\alpha=\alpha_{2}$, then

Proposition 3: For the continuous action rational inattention decision problem, given a binary prior distribution, no matter what forms of the utility function takes, the optimal consideration set and the optimal action strategies are same thing.

Because the optimal consideration set and optimal action strategies are the same thing, therefore if we want to identify $\alpha_{1}$ and $\alpha_{2}$, we can either use $F(\alpha)=1$ of $\alpha=\alpha_{1}$ or $\alpha_{2}$ or use the optimal action strategy. However, $q$ is unknown

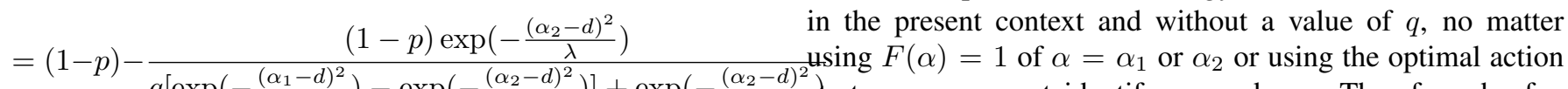
strategy, we cannot identify $\alpha_{1}$ and $\alpha_{2}$. Therefore, by far, in existing literature, at least for rational inattention decision models with binary prior distributions, no such models can be

which can be reformulated to

$$
\begin{gathered}
\frac{-p q\left[\exp \left(-\frac{\left(\alpha_{1}-u\right)^{2}}{\lambda}\right)-\exp \left(-\frac{\left(\alpha_{2}-u\right)^{2}}{\lambda}\right)\right]}{q\left[\exp \left(-\frac{\left(\alpha_{1}-u\right)^{2}}{\lambda}\right)-\exp \left(-\frac{\left(\alpha_{2}-u\right)^{2}}{\lambda}\right)\right]+\exp \left(-\frac{\left(\alpha_{2}-u\right)^{2}}{\lambda}\right)} \\
=-\frac{(1-p) q\left[\exp \left(-\frac{\left(\alpha_{1}-d\right)^{2}}{\lambda}\right)-\exp \left(-\frac{\left(\alpha_{2}-d\right)^{2}}{\lambda}\right)\right]}{q\left[\exp \left(-\frac{\left(\alpha_{1}-d\right)^{2}}{\lambda}\right)-\exp \left(-\frac{\left(\alpha_{2}-d\right)^{2}}{\lambda}\right)\right]+\exp \left(-\frac{\left(\alpha_{2}-d\right)^{2}}{\lambda}\right)}
\end{gathered}
$$

We do not consider the corner solution $q=0$ here. Therefore, the above equation can be reformulated by

$$
\begin{aligned}
q\left[\exp \left(-\frac{\left(\alpha_{1}-u\right)^{2}}{\lambda}\right)\right. & \left.-\exp \left(-\frac{\left(\alpha_{2}-u\right)^{2}}{\lambda}\right)\right]\left[\exp \left(-\frac{\left(\alpha_{1}-d\right)^{2}}{\lambda}\right)\right. \\
& \left.-\exp \left(-\frac{\left(\alpha_{2}-d\right)^{2}}{\lambda}\right)\right]
\end{aligned}
$$$$
=-p \exp \left(-\frac{\left(\alpha_{2}-d\right)^{2}}{\lambda}\right)\left[\exp \left(-\frac{\left(\alpha_{1}-u\right)^{2}}{\lambda}\right)-\exp \left(-\frac{\left(\alpha_{2}-u\right)^{2}}{\lambda}\right)\right]
$$

\section{The Probability Determined by Opti- mal Actions should Follow an Exoge- nous Probability Distribution.}

In last section, we have seen that for the continuousaction rational inattention decision model, the model itself is unidentifiable. It means for the optimal consideration set, it can be any $\left(q, \alpha_{1}, \alpha_{2}\right)$ that follow $q=v \times 1\{0 \leq v \leq 1\}+0 \times\{v<0\}+1 \times\left\{\begin{array}{c}v>p \\ 1-p\end{array}\right\}$ where $v=\frac{p}{1-\exp \left(\frac{-\left(\alpha_{1}-d\right)^{2}+\left(\alpha_{2}-d\right)^{2}}{\lambda}\right)}+\frac{1-p}{1-\exp \left(\frac{-\left(\alpha_{1}-u\right)^{2}+\left(\alpha_{2}-u\right)^{2}}{\lambda}\right)}$. Therefore, no matter whether it is $\alpha_{1}$ or $\alpha_{2}$, they can take any value of real number.

$-(1-p) \exp \left(-\frac{\left(\alpha_{2}-u\right)^{2}}{\lambda}\right)\left[\exp \left(-\frac{\left(\alpha_{1}-d\right)^{2}}{\lambda}\right)-\exp \left(-\frac{\left(\alpha_{2}-d\right)^{2}}{\lambda}\right.\right.$

$q=v \times 1\{0 \leq v \leq 1\}+0 \times\{v<0\}+1 \times\{v>1\}$ is not decessarily a probability. By definition, $q$ is the probability of choosing $\alpha_{1}$. For example, given $\alpha_{2}$, we can find that $q$ is not monotonic with respect to $\alpha_{1}$ and $\lim _{\alpha_{1} \rightarrow-\infty} q \neq 0$. Hence, $q$ cannot be a probability distribution function. Therefore, in fact, not any $\alpha_{1}$ and $\alpha_{2}$ can make function $q$ as a probability. The $\alpha_{1}$ and $\alpha_{2}$ that make $q$ as a probability must satisfy the fundamental properties of probability, which means $\alpha_{1}$ and $q=\frac{p}{1-\exp \left(\frac{-\left(\alpha_{1}-d\right)^{2}+\left(\alpha_{2}-d\right)^{2}}{\lambda}\right)}+\frac{1-p}{1-\exp \left(\frac{-\left(\alpha_{1}-u\right)^{2}+\left(\alpha_{2}-u\right)^{2}}{\lambda}\right)} \begin{aligned} & \alpha_{2} \text { also follow an exogenous probability function and the } \\ & \text { probability taken at } \alpha_{1} \text { and } \alpha_{2} \text { according to the exogenous }\end{aligned}$ 
probability function just equals to $q$ and $1-q$ respectively, i.e.

$\left\{\begin{array}{c}F\left(\alpha_{1} \mid \alpha_{2}\right)=v \times 1\{0 \leq v \leq 1\}+0 \times\{v<0\}+1 \times\{v>1\} \\ F\left(\alpha_{2} \mid \alpha_{1}\right)=(1-v \times 1\{0 \leq 1-v \leq 1\})+0 \times\{1-v<0\} \\ +1 \times\{1-v>1\}\end{array}\right.$

where $F\left(\alpha_{1} \mid \alpha_{2}\right)$ is an exogenous probability function with respect to $\alpha_{1}$ given $\alpha_{2}$ and $F\left(\alpha_{2} \mid \alpha_{1}\right)$ is an exogenous probability function with respect to $\alpha_{2}$ given $\alpha_{1}$. According to equation group (4), it is no longer that any value of $\alpha_{1}$ and $\alpha_{2}$ in the real number set can be regarded as the elements in the optimal consideration set. For example, a numerical example shows that when the exogenous distribution follows a Logit distribution $F(\alpha)=\frac{1}{1+\exp (-\alpha)}$ where $\alpha_{1}$ and $\alpha_{2}$ are independent from each other, and $d=1, u=10, \lambda=0.3, p=0.3$, we can obtain the following two solutions from equation (4) as the elements in the optimal consideration set. They are $\alpha_{1}=-0.847, \alpha_{2}=3.847$ and $q=0.3$, and $\alpha_{1}=1.758$, $\alpha_{2}=1.242$ and $q=0.853$ respectively. However, we want to ask in the general sense, whether equation group (4) can always guarantee a solution and how many solutions there is in the equation group. In the following, for simplicity, let us assume that $\alpha_{1}$ and $\alpha_{2}$ are independent from each other, and the distribution function $F(\alpha)$ satisfies the following properties: $F(\alpha) \in(0,1) \forall \alpha \in(-\infty,+\infty), F(-\infty)=0$ and $F(+\infty)=1$. Therefore, accordingly, equation (4) can be transformed to the following form:

$\left\{\begin{array}{l}F\left(\alpha_{1}\right)=\frac{p}{1-\exp \left(\frac{-\left(\alpha_{1}-d\right)^{2}+\left(\alpha_{2}-d\right)^{2}}{\lambda}\right)}+\frac{1-p}{1-\exp \left(\frac{-\left(\alpha_{1}-u\right)^{2}+\left(\alpha_{2}-u\right)^{2}}{p^{\lambda}}\right)} \\ F\left(\alpha_{2}\right)=\frac{1-p^{\lambda}}{1-\exp \left(\frac{-\left(\alpha_{2}-d\right)^{2}+\left(\alpha_{1}-d\right)^{2}}{\lambda}\right)}+\frac{1}{1-\exp \left(\frac{-\left(\alpha_{2}-u\right)^{2}+\left(\alpha_{1}-u\right)^{2}}{\lambda}\right)}\end{array}\right.$

Observing the two equations in equation group (5), they are essentially symmetric around $\alpha_{2}=\alpha_{1}$. From the first equation in (5), we can get a relationship between $\alpha_{1}$ and $\alpha_{2}$ and we denote it by $\alpha_{1}=g\left(\alpha_{2}\right)$. According to symmetry, $\alpha_{2}=g\left(\alpha_{1}\right)$ satisfies the second equation in equation group (5). What we are looking for is the intersection points between $\alpha_{1}=g\left(\alpha_{2}\right)$ and $\alpha_{2}=g\left(\alpha_{1}\right)$. Because $\alpha_{1}=g\left(\alpha_{2}\right)$ and $\alpha_{2}=g\left(\alpha_{1}\right)$ are symmetric around $\alpha_{2}=\alpha_{1}$, therefore the intersection points between the two functions, if they have, should located on the line $\alpha_{2}=-\alpha_{1}+k$ where $k \in \mathbb{R}$ is a constant.

If there are are only two intersection points between $\alpha_{2}=g\left(\alpha_{1}\right)$ and $\alpha_{2}=-\alpha_{1}+k$, then according to symmetry, only when these two points are also the intersection points between $\alpha_{1}=g\left(\alpha_{2}\right)$ and $\alpha_{2}=-\alpha_{1}+k$, then these two points are also the intersection points between $\alpha_{2}=g\left(\alpha_{1}\right)$ and $\alpha_{1}=g\left(\alpha_{2}\right)$ (see Figure 5). However, if the intersection points between $\alpha_{2}=g\left(\alpha_{1}\right)$ and $\alpha_{2}=-\alpha_{1}+k$ are not the intersection points $\alpha_{1}=g\left(\alpha_{2}\right)$ and $\alpha_{2}=-\alpha_{1}+k$, then these two points are not the intersection points between $\alpha_{2}=g\left(\alpha_{1}\right)$ and $\alpha_{1}=g\left(\alpha_{2}\right)$ (see Figure 6).

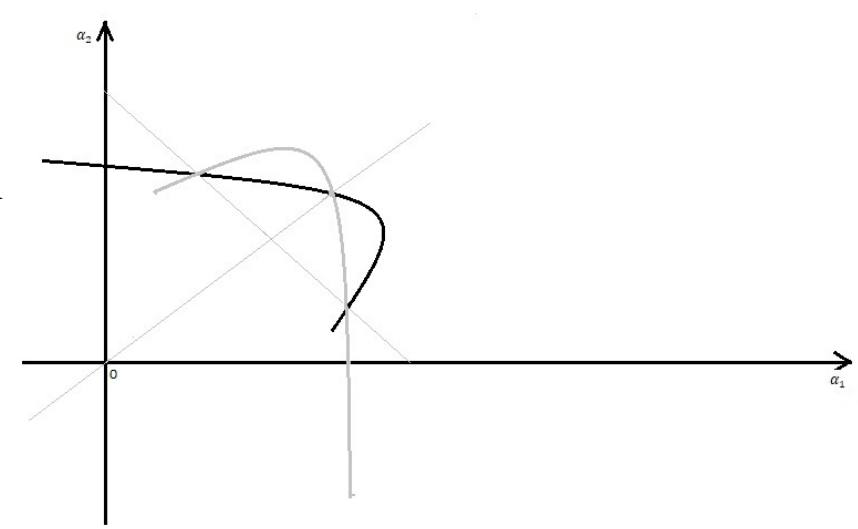

Figure 5: The black curve represents $\alpha_{1}=g\left(\alpha_{2}\right)$. The gray curve represents $\alpha_{2}=g\left(\alpha_{1}\right)$. The thin lines are auxiliary lines, which are $\alpha_{2}=\alpha_{1}$ and $\alpha_{2}=-\alpha_{1}+k$ respectively. This figure shows that if $\alpha_{2}=g\left(\alpha_{1}\right)$ and $\alpha_{2}=-\alpha_{1}+k$ have two intersection points, then the two intersection points must also be the intersection points between $\alpha_{2}=g\left(\alpha_{1}\right)$ and $\alpha_{1}=g\left(\alpha_{2}\right)$. The two intersection points are also the solutions to equation group (5).

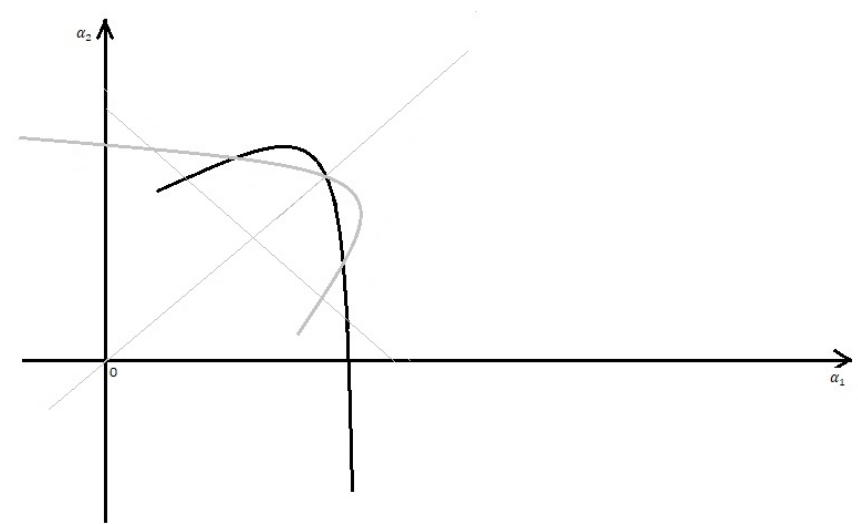

Figure 6: The black curve represents $\alpha_{2}=g\left(\alpha_{1}\right)$. The gray curve represents $\alpha_{1}=g\left(\alpha_{2}\right)$. The thin lines are auxiliary lines, which are $\alpha_{2}=\alpha_{1}$ and $\alpha_{2}=-\alpha_{1}+k$ respectively. This figure shows that if $\alpha_{2}=g\left(\alpha_{1}\right)$ and $\alpha_{2}=-\alpha_{1}+k$ have two intersection points, but the two intersection points are not the intersection points between $\alpha_{1}=g\left(\alpha_{2}\right)$ and $\alpha_{2}=-\alpha_{1}+k$, then the two intersection points are not the intersection points between $\alpha_{2}=g\left(\alpha_{1}\right)$ and $\alpha_{1}=g\left(\alpha_{2}\right)$ as well. In this situation, the solutions cannot be found for equation group (5)

Proposition 4: For the binary state rational inattention continuous-action decision problem with quadratic utility function, if and only if the intersection points between $\alpha_{2}=-\alpha_{1}+k$ and $\alpha_{1}=g\left(\alpha_{2}\right)$ and the intersection points between $\alpha_{2}=-\alpha_{1}+k$ and $\alpha_{2}=g\left(\alpha_{1}\right)$ are the same, then these intersection points are the intersection points between $\alpha_{1}=g\left(\alpha_{2}\right)$ and $\alpha_{2}=g\left(\alpha_{1}\right)$.

Under our specification where $F(-\infty)=0$ and $F(+\infty)=1$, equation group (5) will not have a solu- 
tion where $\alpha_{2}=\alpha_{1}=\frac{k}{2}$. The reason is that $\alpha_{2}=\alpha_{1}=\frac{k}{2}$ are discontinuity points for $1-v$ with respect to $\alpha_{2}$ and $v$ with respect to $\alpha_{1}$. At this point, function $q$ reaches corner solutions where $q=1$ or 0 . Therefore, as long as $\frac{k}{2} \in(-\infty,+\infty), F\left(\alpha_{1}\right)$ and $q$ and hence $v$ will not have any intersection points, so do $F\left(\alpha_{2}\right)$ and $1-q$ and hence $1-v$.

In the following situations, equation group (5) would never have any solutions.

\subsection{The situation where equation group would never have any solutions}

(5)

If either equation in equation group (5) and $\alpha_{2}=-\alpha_{1}+k$ has no intersection points, then according to symmetry, the other equation in equation group (5) does not have any intersection with $\alpha_{2}=-\alpha_{1}+k$ as well. In this situation, the equation group has no solutions. In this section, we will show that when $k>2 u$ or $k<2 d$, equation group (5) has no solutions.

Substituting $\alpha_{2}=-\alpha_{1}+k$ into $v, v$ can be transformed to the following formula:

$$
v=\frac{p}{1-\exp \left(\frac{\left(2 \alpha_{1}-k\right)(2 d-k)}{\lambda}\right)}+\frac{1-p}{1-\exp \left(\frac{\left(2 \alpha_{1}-k\right)(2 u-k)}{\lambda}\right)}
$$

It can be observed that $v$ is discontinuous at $\alpha_{1}=\frac{k}{2}$. Further,

$$
\begin{gathered}
\frac{\partial v}{\partial \alpha_{1}}=\frac{p \exp \left(\frac{\left(2 \alpha_{1}-k\right)(2 d-k)}{\lambda}\right)}{\left[1-\exp \left(\frac{\left(2 \alpha_{1}-k\right)(2 d-k)}{\lambda}\right)\right]^{2}} \frac{2(2 d-k)}{\lambda} \\
+\frac{(1-p) \exp \left(\frac{\left(2 \alpha_{1}-k\right)(2 u-k)}{\lambda}\right)}{\left[1-\exp \left(\frac{\left(2 \alpha_{1}-k\right)(2 u-k)}{\lambda}\right)\right]^{2}} \frac{2(2 u-k)}{\lambda}
\end{gathered}
$$

If $k>2 u$, then $\frac{\partial v}{\partial \alpha_{1}}<0 . \lim _{\alpha_{1} \rightarrow+\infty} v=1$ and $\lim _{\alpha_{1} \rightarrow-\infty} v=0$. Therefore, in this situation, $v$ can be expressed by the following figure (see Figure 7).

According to our specification, $F(\alpha) \in(0,1)$ for $\alpha \in(-\infty,+\infty)$. Therefore, in this situation, $F\left(\alpha_{1}\right) \neq v$ for all $\alpha \in(-\infty,+\infty)$. The same situation applies to $F\left(\alpha_{2}\right) \neq 1-v$ according to symmetry. Therefore, equation group (5) does not have any solution for $k>2 u$.

Now consider the situation where $k<2 d$. In this situation, $\frac{\partial v}{\partial \alpha_{1}}>0, \lim _{\alpha_{1} \rightarrow+\infty} v=0$, and $\lim _{\alpha_{1} \rightarrow-\infty} v=1$ given that $\alpha_{2}=-\alpha_{1}+k$. Therefore, in this situation, $v$ can be described by the following figure (see Figure 8).

According to Figure 8, we can see that for $k<2 d$, probability distribution function $F\left(\alpha_{1}\right)$ still cannot intersect $v$ for $-\infty<\alpha_{1}<+\infty$. Therefore, in this situation, according to

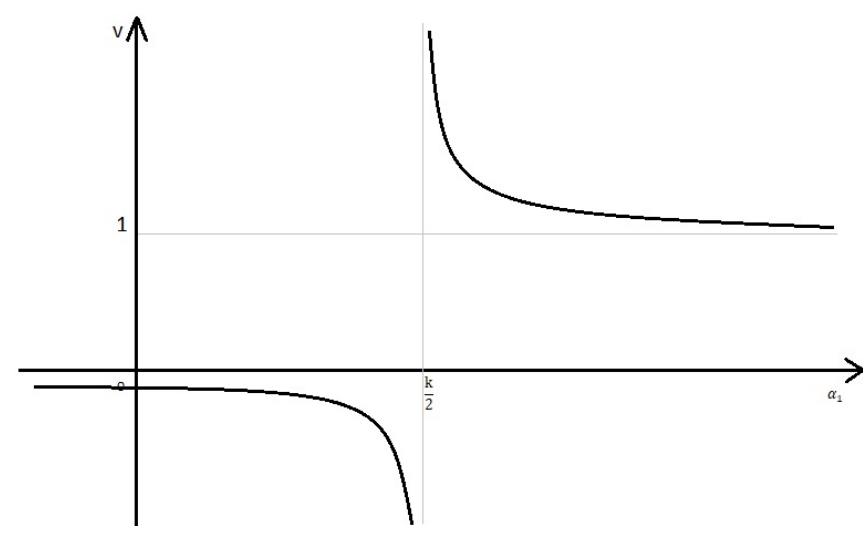

Figure 7: The function $v$ given $\alpha_{2}=-\alpha_{1}+k$ where $k>2 u$.

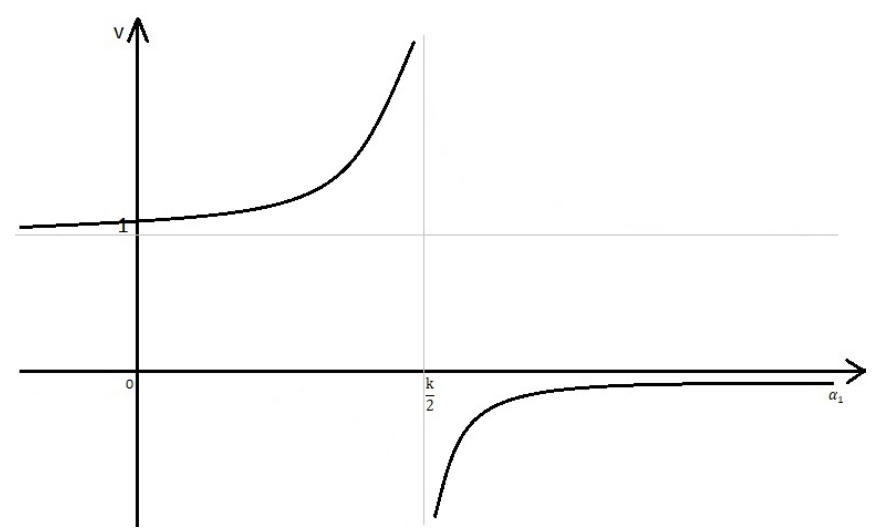

Figure 8: The function $v$ given $\alpha_{2}=-\alpha_{1}+k$ where $k<2 d$.

the same reasons as in the case where $k>2 u$, equation group (5) has no solutions.

For $k=2 d$ or $k=2 u, v=+\infty$ or $-\infty$ and hence $q=1$ or 0 given $\alpha_{2}=-\alpha_{1}+k$ for all $\alpha_{1} \in \mathbb{R}$. However, since we have assumed that only when $\alpha=+\infty$ or $-\infty, F(\alpha)=1$ or 0 . Therefore, in our discussion, when $k=2 d$ or $k=2 u$, equation group (5) still do not have solutions.

\subsection{For $2 d<k<2 u$, the solutions exist and the number of them are infinite. Equation group (5) in fact does not describe scatter points but it describes a curve or curves of $\alpha_{2}$ with respect to $\alpha_{1}$.}

First, we prove that given $\alpha_{2}=-\alpha_{1}+k, \frac{\partial v}{\partial \alpha_{1}}<0$ for $\alpha_{1} \in\left(-\infty, \frac{k}{2}\right)$ or $\alpha_{1} \in\left(\frac{k}{2},+\infty\right)$. In the following, for convenience, we call the function $v$ given $\alpha_{2}=-\alpha_{1}+k$ by simply $v$.

Define $y(x)=\frac{x \exp (x)}{(1-\exp (x))^{2}}, x_{2}=\frac{\left(2 \alpha_{1}-k\right)(2 d-k)}{\lambda}$ and $x_{1}=\frac{\left(2 \alpha_{1}-k\right)(2 u-k)}{\lambda}$. Therefore, $\frac{\partial v}{\partial \alpha_{1}}\left(2 \alpha_{1}-k\right)=$ $p y\left(x_{2}\right) \times 2+(1-p) y\left(x_{1}\right) \times 2 . \quad y(x)$ is discontinuous at $x=0 . \lim _{x \rightarrow+\infty} y(x)=0$ and $\lim _{x \rightarrow-\infty} y(x)=0$. It decreases with respect to $x \in(-\infty, 0)$ or $x \in(0,+\infty) . y(x)$ 
is also symmetric around $(0,0)$. The function can be expressed by the following figure (see Figure 9).

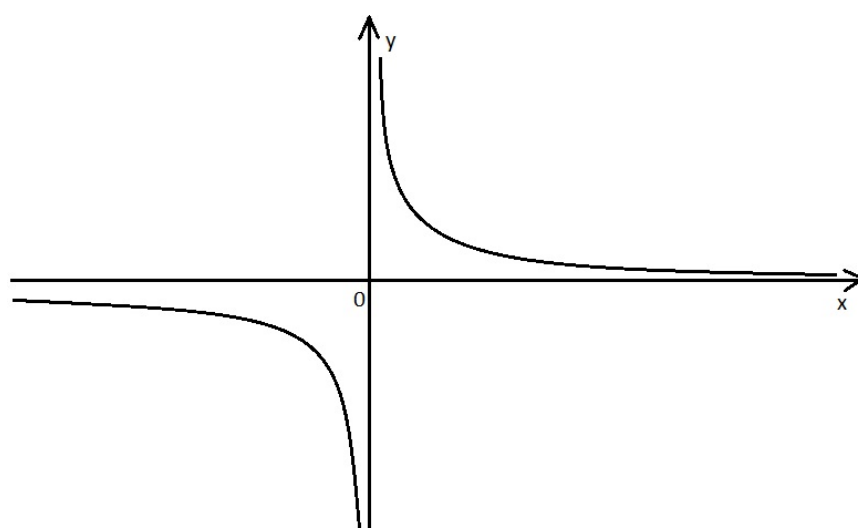

Figure 9: Function $y(x)=\frac{x \exp (x)}{(1-\exp (x))^{2}}$

Consider $\alpha_{1}>\frac{k}{2}$. Because $2 d<k<2 u, x_{1}>0$ and $x_{2}<0$. According to symmetry, if $x_{2}>-x_{1}$, then $-y\left(x_{1}\right)>y\left(x_{2}\right) . x_{2}>-x_{1}$ can be reformulated to $k<u+d$. Therefore, in this situation, if $p>0.5$, then $\frac{\partial v}{\partial \alpha_{1}}<0$. According to symmetry, for $\alpha_{1}<\frac{k}{2}$, if $p>0.5$, as long as $k<u+d$, then $\frac{\partial v}{\partial \alpha_{1}}<0$. Therefore, for $p>0.5$ and $k<u+d, \frac{\partial v}{\partial \alpha_{1}}<0$ for $\alpha_{1} \in\left(-\infty, \frac{k}{2}\right)$ or $\alpha_{1} \in\left(\frac{k}{2},+\infty\right)$.

Lemma 1: Given $\alpha_{2}=-\alpha_{1}+k$, for $p>0.5$ and $2 d<k<u+d, \frac{\partial v}{\partial \alpha_{1}}<0$ for $\alpha_{1} \in\left(-\infty, \frac{k}{2}\right)$ or $\alpha_{1} \in\left(\frac{k}{2},+\infty\right)$.

Given that $2 d<k<u+d, \lim _{\alpha_{1} \rightarrow-\infty} v=1-p$ and $\lim _{\alpha_{1} \rightarrow-\infty} v=p$. Besides, because $v$ is monotonic and discontinuous at $\alpha_{1}=\frac{k}{2}$, for $\alpha_{1}<\frac{k}{2}$, there exists a unique $\alpha_{1}$ such that $v=0$. Let's denote the $\alpha$ by $\alpha_{1}(0)$. For $\alpha \in\left(\alpha_{1}(0), \frac{k}{2}\right), v<0$ and therefore in this area, $q$ reaches corner solution and it equals 0 . Similarly, for $\alpha_{1}>\frac{k}{2}$, there exists a unique $\alpha_{1}$ such that $v=1$. Let's denote the $\alpha$ by $\alpha_{1}(1)$. For $\alpha \in\left(\frac{k}{2}, \alpha_{1}(1)\right), v>1$ and therefore in this area, $q$ reaches corner solution and it equals 1 . Equation $v$ and equation $q$ can be reflected by the following figure (see Figure 10).

For $\alpha_{1}=-\infty$, as we have demonstrated, $v=1-p>$ $0=\lim _{\alpha_{1} \rightarrow-\infty} F\left(\alpha_{1}\right)$. For $\alpha_{1}=\frac{k}{2}-\varepsilon$ where $\varepsilon$ is an arbitrarily small positive number, $v=0<F\left(\alpha_{1}\right)$. Remember that $F\left(\alpha_{1}\right)$ increases and $q$ decreases with respect to $\alpha_{1}$ for $\alpha_{1}<\frac{k}{2}$. Therefore, for $\alpha_{1}<\frac{k}{2}$, there exists a unique intersection point between $F\left(\alpha_{1}\right)$ and $q$ and at this point $q \in(0,1)$, which means the point can also be regarded as the intersection point between $F\left(\alpha_{1}\right)$ and $v$.

For $\alpha_{1}=+\infty$, as we have demonstrated, $v=p<1=$ $\lim _{\alpha_{1} \rightarrow+\infty} F\left(\alpha_{1}\right)$. For $\alpha_{1}=\frac{k}{2}+\varepsilon$ where $\varepsilon$ is an arbitrarily small positive number, $v=1>F\left(\alpha_{1}\right)$. Still, $F\left(\alpha_{1}\right)$ increases and $q$ decreases with respect to $\alpha_{1}$ for $\alpha_{1}>\frac{k}{2}$. Therefore, for $\alpha_{1}>\frac{k}{2}$, there exists a unique intersection point between

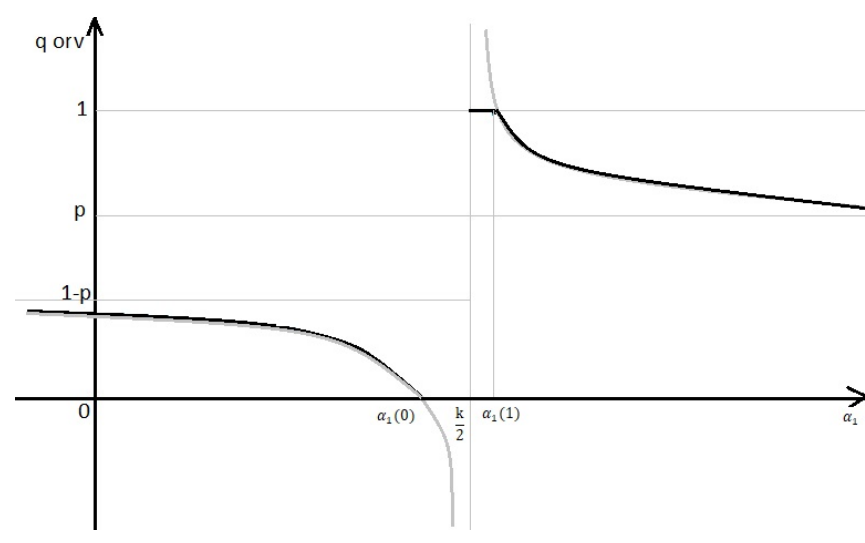

Figure 10: The gray curve depicts equation $v$ and the black curve depicts equation $q$. The thin lines are auxiliary curves.

$F\left(\alpha_{1}\right)$ and $q$ and at this point $q \in(0,1)$, which means the point can also be regarded as the intersection point between $F\left(\alpha_{1}\right)$ and $v$.

Therefore, in total, given a $k \in(2 d, u+d), F\left(\alpha_{1}\right)$ and $v$ have two intersection points.

Lemma 2: If $p>0.5$, given a $k \in(2 d, u+d), F\left(\alpha_{1}\right)$ and $v$ have two intersection points.

Given $\alpha_{2}=-\alpha_{1}+k$, it is found that $v$ and $1-v$ are symmetric around $\left(\frac{k}{2}, \frac{1}{2}\right)$. Because $F\left(\alpha_{1}\right)$ and $F\left(\alpha_{2}\right)=1-F\left(\alpha_{1}\right)$ are also symmetric around $y=\frac{1}{2}$, therefore the intersection points of $F\left(\alpha_{1}\right)$ and $v$ and of $F\left(\alpha_{2}\right)$ and $1-v$ actually correspond to each other: if $F\left(\alpha_{1}\right)$ takes an intersection point with $v$ at an $\alpha_{1}$, then at the same $\alpha_{1}, F\left(\alpha_{2}\right)$ and $1-v$ take another intersection point, and vice versa. Besides, the corresponding $F\left(\alpha_{1}\right)$ and $F\left(\alpha_{2}\right)$ satisfies $F\left(\alpha_{1}\right)+F\left(\alpha_{2}\right)=1$. Therefore, in equation group (5), as long as we are discussing the intersection points between $F\left(\alpha_{1}\right)$ and $v$, we are at the mean time discussing the intersection points between $F\left(\alpha_{2}\right)$ and $1-v$. Further, this result implies that in equation group (5), the intersection points between $\alpha_{2}=-\alpha_{1}+k$ and $\alpha_{1}=g\left(\alpha_{2}\right)$ (the first equation in equation group (5)) are exactly the intersection points between $\alpha_{1}=g\left(\alpha_{2}\right)$ and $\alpha_{2}=g\left(\alpha_{1}\right)$ (the second equation in equation group (5)).

Lemma 3: Given $k \in(2 d, u+d)$, the intersection points between $\alpha_{2}=-\alpha_{1}+k$ and the first (second) equation of equation group (5) are the intersection points between the two equations of equation group (5).

According to Lemma 3, Lemma 2 can be further written into the following results:

Proposition 5: If $p>0.5$, given a $k \in(2 d, u+d)$, equation group (5) have two intersection points.

Therefore, based on Proposition 5, we obtain a critical result that fundamentally matters for the binary state continuousaction rational inattention decision problem. 
Corollary 1: Supposing the prior distribution is binary and the exogenous probability distribution $F(\alpha)$ satisfies our pre-specified assumptions, equation group (5) have solutions.

However, note that for any $k \in(2 d, u+d)$, equation group (5) can always return a solution. Therefore, given all exogenous parameters, equation group (5) can result in infinite solutions.

Proposition 6: Supposing the prior distribution is binary and the exogenous probability distribution $F(\alpha)$ satisfies our pre-specified assumptions, the number of the solutions of equation group (5) is infinite.

Therefore, equation group (5) does not describe several scatter points of $\left(\alpha_{1}, \alpha_{2}\right)$, but describes a curve or curves of $\alpha_{2}$ with respect to $\alpha_{1}$, which can be called the optimal solution curve(s). For $k \in(2 d, u+d)$, we can be certain about the properties of the curve. For example, given $k \in(2 d, u+d)$, if we draw a line $\alpha_{2}=-\alpha_{1}+k$, it must intersect the optimal solution curve with two intersection points and the two intersection points are located on both sides around $\alpha_{2}=\alpha_{1}$. Because equation group (5) reflects the optimal consideration set, our results can guarantee that at least a subset of the optimal consideration set is a curve or curves. The reason why we say it is a subset is that by far we have studied cases where $2 d<k<u+d$, and we have not studied cases where $u+d \leq k<2 u$. Interested readers can follow our approach in this paper to explore the optimal consideration set where $u+d \leq k<2 u$.

Lemma 4: In the binary state continuous-action rational inattention decision problem with quadratic utility function $-(\alpha-\varepsilon)^{2}$, a subset of the optimal consideration set is a curve or curves of $\alpha_{2}$ with respect to $\alpha_{1}$.

\section{Conclusion}

We obtain three results by analyzing the binary state continuous-action rational inattention decision problems. The first result is that given the binary state, it is the form of the utility function that determines the form of the posterior distribution, i.e. whether it is a binary distribution or triple or quadruple distribution, etc. The second result is that for the binary state continuous-action rational inattention decision problems, the optimal consideration set is essentially the optimal action strategies, which makes the model unidentifiable. The third result is that if we want to identify the model, we need extra condition. A natural condition arises such that the optimal actions in the optimal consideration set should also satisfy an exogenously-specified probability distribution. We test this idea by adopting the quadratic utility function in the rational inattention decision problem, and we find that with this condition, the model is identified. Because under quadratic utility function, the posterior exhibits a binary form, the optimal consideration set identified is a curve or curves of the two discrete signals, in which one signal or action is a function of another. We believe that the three results can be extrapolated to more general form of continuous-action rational inattention decision problems, e.g. the prior distribution follows Lebesgue measure. Our purpose in this paper is to provide some insights through simple examples into deepening readers' understanding towards continuous-action rational inattention decision problems, and we hope we have achieved this purpose in this paper.

For the future extensions of the research in our paper, we point out three directions that we will address:

1. In this paper, we have managed to identify a subset of the optimal consideration set. In future research, by studying the same model (i.e., the continuous-action rational inattention decision model of quadratic utility function with binary prior distribution), we can attempt to identify the complete optimal consideration set;

2. After studying the first direction, we can move on to study the theory and econometrics of continuous-action rational inattention decision models with general utility functions or general prior distributions.

3. We will also attempt the estimation of the optimal consideration set in future research.

Rational inattention theory is a promising field that links economic theory and neurosciences (Mikhael, Lai, Gershman [9]; Wang [15]). The researches on econometrics of rational inattention theory are emerging. It can be expected that the discoveries in this area will boom in the foreseeable future.

\section{REFERENCES}

[1] Caplin A., Dean M., Leahy J., "Rational Inattention, Optimal Consideration Sets, and Stochastic Choice," Review of Economic Studies, vol. 86, no. 3, pp. 1061-1094, 2019. https://doi.org/10.1093/restud/rdy037.

[2] Cover T., Thomas J., "Elements of Information Theory," 2nd ed, John Wiley \& Sons, Inc., 2006.

[3] Jung J., Kim J. H., Matějka F., Sims C. A., "Discrete Actions in Information-Constrained Decision Problems," Review of Economic Studies, vol. 86, no. 6, pp. 2643-2667, 2019. https://doi.org/10.1093/restud/rdz011.

[4] Kahneman D., "Attention and Effort," Prentice-Hall, Inc., Englewood Cliffs, New Jersey, 1973. 
[5] Lachman R., Lachman J. L., Butterfield B. C., "Cognitive Psychology and Information Processing: an Introduction," Psychology Press, Taylor \& Francis Group, London and New York, 1979, reprinted in 2015.

[6] Maćkowiak B., Matějka F., Wiederholt M., "Rational Inattention: a Review,” Working Paper, CERGE-EI, 2021.

[7] Matějka F., McKay A., "Rational Inattention to Discrete Choices: a New Foundation for the Multinomial Logit Model," American Economic Review, vol. 105, no. 1, pp. 272-298, 2015.

[8] Matějka F., "Rationally Inattentive Seller: Sales and Discrete Pricing," Review of Economic Studies, vol. 83, no. 3, pp. 1125-1155, 2016. https://doi.org/10.1093/restud/rdv049.

[9] Mikhael J., Lai L., Gershman S., "Rational Inattention and Tonic Dopamine," PLoS Computational Biology, vol. 17, no. 3: e1008659, 2021. https://doi.org/10.1371/journal.pcbi.1008659.

[10] Shannon C. E., "A Mathematical Theory of Communication," The Bell System Technical Journal, vol. 27, no. 3, pp. 379-423, 623-656, 1948. https://doi.org/10.1002/j.15387305.1948.tb01338.x.

[11] Sims C. A., "Stickiness," Carnegie-Rochester Conference Series on Public Policy, vol. 49, pp. 317-356, 1998.

[12] Sims C. A., "Implications of Rational Inattention," Journal of Monetary Economics, vol. 50, no. 3, pp. 665-690, 2003.

[13] Sims C. A., "Rational Inattention: A Research Agenda," Working Paper, Princeton University, 2006.

[14] Tutino A., “'Rational Inattention' guides overloaded brains, help economists understand market behavior," Economic Letter, Federal Reserve Bank of Dallas, vol. 6 (Mar), 2011.

[15] Wang R., “The Rationally Inattentive Manager," Advances in Economics and Business, vol. 8, no. 1, pp. 39 - 62, 2020. DOI: 10.13189/aeb.2020.080105. 